\title{
Epidemiology of Pancreatic and Periampullary Cancer
}

\author{
Prabin Thapa ${ }^{1}$
}

Received: 24 September 2015 / Accepted: 30 September 2015 / Published online: 17 October 2015

(C) Association of Surgeons of India 2015

\begin{abstract}
Pancreatic cancer is an important public health problem, although infrequent but with an exceptionally high mortality rate worldwide. Given that the majority of cancers occur in association with smoking, diabetes, pancreatitis, genetic factors, and others and with a growing population worldwide in mind, more cases would be expected in the near future giving further impetus to investigating prevention and treatment strategies to this international issue. The representative data on epidemiology of carcinoma of pancreas in India and Nepal are very poor. While there are many questions to be resolved, it is apparent that many facets of pancreatic cancer are becoming increasingly understood, and prospects for prevention are becoming apparent. Hence, screening research, recommendations, and implementation is an obvious priority. Although previous studies have contributed to the knowledge of carcinoma of pancreas epidemiology, such association needs to be further verified with proper epidemiological work. A new and global approach to the study of carcinoma of pancreas epidemiology is required if the disease prevention and treatment strategies are to be adequately directed and supported in the coming years. The collection and analysis of epidemiologic carcinoma of pancreas data will play a critical role in guiding future disease prevention strategies and optimizing patient management.
\end{abstract}

Keywords Carcinoma of pancreas $\cdot$ Epidemiology $\cdot$ Risk factors

Prabin Thapa

prabinbt@gmail.com

1 Department of Surgery, Kathmandu Medical College Teaching Hospital, Sinamangal, Kathmandu, Nepal

\section{Introduction}

Worldwide, carcinoma of the pancreas causes more than a quarter of a million deaths annually, being the 13th most common cancer, the eighth most frequent cause of death from cancer. Survival rates are among the worst for any tumor, being the mortality to incidence ratio of $98 \%$. It is among the tumors with worst survival rates [1].

Resectional surgery does lead to about a $20 \% 5$-year survival, but because of the presence of widespread local disease or metastasis, only $10-20 \%$ of patients undergo pancreatic resection. As current therapeutic results are so dismal, it is essential to review the epidemiologic factors that influence the development of this disease in order to implement preventive and screening strategies that can reduce the burden of this lethal cancer [2].

With the exception of the small bowel, the pancreas is less likely to develop cancer than any other gastrointestinal organ. Nevertheless, the remote location of the organ, the lack of any specific diagnostic markers, the difficulty in establishing a tissue diagnosis, and the aggressive nature of pancreatic adenocarcinomas, which respond poorly to both chemotherapy and radiotherapy, contribute to the exceptionally high mortality associated with this type of cancer. At present, inherited disorders explain only a small part of the total burden of pancreatic cancer. Environmental factors seem to account for a large proportion of pancreatic cancer cases. Known preventive strategies are limited to reducing smoking exposure and maintaining normal body weight. Consuming adequate quantities of fruits and vegetables might also have a preventive effect, although strong epidemiological evidence is lacking. Promising areas for additional research include the substantial racial differences in pancreatic cancer frequency, the surprising link with blood groups, the possible relationship of pancreatic cancer to oncogenic viruses, and validation of the many putative 
gene polymorphisms that have been tentatively identified as risk factors.

\section{Global Trends}

According to SEER data based on 2008-2012, the number of new cases of pancreatic cancer was 12.4 per 100,000 men and women per year, and approximately $1.5 \%$ of men and women will be diagnosed with pancreatic cancer at some point during their lifetime [3]. Despite its low incidence in developed countries, pancreatic cancer is associated with poor survival and ranks as the fourth or fifth most common cause of cancer mortality. The incidence of pancreatic cancer in India is $0.5-$ 2.4 per 100,000 men and $0.2-1.8$ per 100,000 women [4]. Recent data shows a decreasing trend in both incidence and mortality of pancreatic cancer in the UK, though a study done in over 51 countries across the world (Europe, 33 countries; America, 8 countries; and Asia, 10 countries) between 1992 and 2002 revealed that the age-standardized mortality (world) rates (ASR (W)) for pancreatic cancer have been static [5].

\section{Family History and Genetic Disorders (Are Some People Genetically More Prone to Pancreatic Carcinoma?)}

A family history of pancreatic cancer in a first-degree relative is associated with an increased risk of pancreatic cancer of between 2.5 and 5.3 times. The risk increases as more relatives are affected. The increased risk in family members could be owing to genetic factors and/or lifestyle habits that may be similar in different generations, such as smoking. Genetic predisposition is regarded as one of the major risk factors in the development of cancer. The development of pancreatic cancer may be associated with inherited mutations in specific genes. These mutations are therefore the cause of various familial cancer syndromes. These germline disorders account for $5-10 \%$ of pancreatic cancer cases. It is considered that activation of the oncogene K-ras with simultaneous inactivation of the tumor suppressor genes (p53, DPC4, p16, and BRCA2) results in pancreatic cancer. Ninety percent of all cases of pancreatic cancer have p16 mutations, $75 \%$ have p53 mutations, and $55 \%$ have DPC4 mutations. Of the remaining sporadic pancreatic cancers, some could be attributable to somatic polymorphic mutations in genes encoding tobacco and food metabolizing enzymes, as well as to DNA repair genes. A small fraction may evolve due to the dysfunction of the various DNA mismatch repair genes (microsatellite instability (MSI)) [5].

A number of genetic disorders are associated with an increased risk of pancreatic cancer, although no common genetic abnormality has been identified in all these conditions. These include hereditary pancreatitis, familial adenomatous polyposis, familial atypical multiple mole, melanoma, Peutz-Jeghers syndrome, hereditary non-polyposis colorectal cancer, familial breast cancer, ataxia-telangiectasia, and cystic fibrosis [6]. A paternal inheritance pattern and long duration of onset of hereditary pancreatitis have shown to have higher risk [7] and those with Peutz-Jeghers syndrome the relative risk is very high for gastrointestinal and other non-gastrointestinal tumors [8].

\section{Diabetes}

Diabetes mellitus (DM) being a complex metabolic condition defined by the level of hyperglycemia giving rise to risk of microvascular or macrovascular complications [9]. Diabetes is common in Nepal and India. The prevalence in India is around $10 \%$ i.e., 31 million people which is predicted to increase to 80 million by year 2030 . Asians have a strong genetic susceptibility for type II diabetes. Recent onset of diabetes can be associated with pancreatic cancer, an early symptom in nearly $75 \%$ of all pancreatic cancer patients [10]. Huxley et al revealed the relationship of therapy for diabetes with carcinoma of pancreas in which they found that Metformin, an oral antidiabetic agent, is associated with a decreased risk of pancreatic cancer, whereas another study showed that insulin and insulin secretagogues increase the risk of pancreatic cancer [11]. Risk of pancreatic cancer increases up to $50 \%$ in patients with long-term disease ( $\geq 10$ years) with type 2 DM [12].

\section{Chronic Pancreatitis}

Chronic pancreatitis has a significant association with pancreatic cancer. A large, retrospective cohort study of patients with at least 5 years of chronic pancreatitis before being diagnosed with pancreatic cancer found a 14-fold increased risk of pancreatic cancer in patients with chronic pancreatitis [13]. A causative role for pancreatitis in carcinoma of pancreas in humans has been strongly suggested based on various similar studies [14]. The degree of association is very strong between rare autosomal dominant hereditary form of pancreatitis, which arises during or soon after childhood, risk being 50 times than that in the general population [15]. However, chronic pancreatitis is rare and is not a main cause of pancreatic cancer [16].

\section{Smoking}

Nearly all studies published since 1966 have shown an association between smoking and pancreatic cancer. The risk of pancreatic cancer is raised by $75 \%$ as compared to nonsmokers, risk persisting minimum of 10 years after cessation of smoking when the risk gradually diminishes to the baseline [17]. Another study that included nearly 13,000 patients with pancreatic cancer showed that current smokers had an odds ratio of 2.2, compared with never smokers, and the odds ratio was 1.2 for ex-smokers. With cessation of smoking, risk gradually decreases but requires more than 10-20 years before the 
smoking-related risk disappears [18]. Pipes, cigars, other tobacco products, and even oral tobacco products increase the risk of pancreatic cancer. Smokeless tobacco contributes to risk probably lower than that of smokers but higher than that of non-tobacco users [19]. Estimated prevalence of smoking is about $30 \%$ in many parts of the world, and the risk of pancreatic cancer is doubled in smokers. Tobacco smoking releases many carcinogens. A recent study examined several detoxifying genes in 455 patients with pancreatic cancer [20]. Variants in genes such as CYP1B1-4390-GG and uridine 5 '-diphosphoglucuronosyltransferase reduced the risk of pancreatic cancer, whereas variants in others, such as GSTM1, increased risk. Little is known about the mechanisms by which these genes affect cancer risk, but their products could affect the inflammatory response, fibrosis, cell proliferation, and other processes involved in carcinogenesis [21]. Various germline mutations, as in hereditary pancreatitis, familial atypical mole-multiple melanoma (FAMMM) syndrome, Li-Fraumeni syndromes, Peutz-Jeghers, cystic fibrosis, and BRCA1/BRCA2, were suggested to increase the risk of early onset pancreatic cancer in interaction with smoking suggesting the primary role of smoking in early onset pancreatic cancer [22].

\section{Age, Gender, and Race}

Age is the strongest risk factor for pancreatic cancer. The risk is almost 20 times higher for individuals older than 50 years as compared to younger persons. As for other cancers, fewer than $10 \%$ of cases of pancreatic cancer occur among individuals younger than 55 years old, and the median age of onset is 71 years. In all groups, men have higher incidence rates than women [23]. Few if any, gender-specific hormonal risk factors seem to have a causal role in pancreatic cancer and that carcinoma of pancreas is an estrogen dependent tumor at least in part. [24] Since smoking rates are higher in men than in women, smoking could be responsible for the higher rates observed in men.

Fifty to ninety percent higher incidence of pancreatic cancer in blacks compared with whites seen for decades is due to established risk factors (mainly cigarette smoking and diabetes) in men and less accepted risk factors (i.e., heavy alcohol drinking and elevated BMI) in women [25].

\section{Alcohol}

Alcohol is a major risk factor for pancreatitis; however, studies have failed to support its association with pancreatic cancer including a recent large retrospective cohort study from Sweden [26] whereas only a weak relation has been reported by some studies [27]. As there is a strong relationship between smoking and alcohol consumption, confounding factor being smoking could be the reason of weak association that could not be excluded.

\section{Diet}

No strong link has been found between diet and pancreatic cancer [28]. However, study done by Ghorbani $\mathrm{Z}$ et al. [29] have shown in a well-matched case control study that increased frequency of intake of rice, bread and barbequed meat and deep fried vegetables can aggregate the risk while consumption of fish can decrease the incidence of pancreatic cancer. There has been no association between intake of fat and risk of pancreatic cancer [30] while exercise, which helps to maintain normal body weight, has been associated with a reduced risk of pancreatic cancer [31]. Non-steroidal anti-inflammatory drugs (NSAIDs), mainly aspirin, have shown to decrease risk of pancreatic cancer [32, 33]; however, other non-aspirin NSAIDs have not shown the benefit [34]. Similarly, lipid-lowering statins have a modest role in decreasing risk of pancreatic cancer [35].

\section{Conclusions}

Type II diabetes, heavy alcohol drinking, cigarette smoking, excess body weight, chronic pancreatitis, a family history of pancreatic cancer, and known few germline mutations are some of the identifiable risk factors for pancreatic cancer. Chronic inflammation in the pancreas due to several reasons like chronic pancreatitis, alcohol, smoking, hyperlipidemia, etc. is probably involved in the development pancreatic cancer.

\section{Compliance with Ethical Standards}

Conflict of Interest The author declares that he has no competing interests.

\section{References}

1. Parkin DM, Bray F, Ferlay J, Pisani P (2002) Global cancer statistics. CA Cancer J Clin 55(2):74-108

2. Raimondi S, Maisonneuve P, Lowenfels AB (2009) Epidemiology of pancreatic cancer: an overview. Nat Rev Gastroenterol Hepatol 6(12):699-708

3. SEER Cancer Statistics Factsheets: Pancreas Cancer. National Cancer Institute. Bethesda, MD, http://seer.cancer.gov/statfacts/ $\mathrm{html} /$ pancreas.html

4. Dhir V, Mohandas K (1999) Epidemiology of digestive tract cancers in India IV. Gall bladder and pancreas. Indian J Gastroenterol 18(1):24-28 
5. Calhoun ES, Kern SE (2008) Molecular genetics of pancreatic cancer. In: Lowy AM, Leach SD, Philip PA (eds) Pancreatic cancer. Springer, New York

6. Hart AR, Kennedy H, Harvey I (2008) Pancreatic cancer: a review of the evidence on causation. Clin Gastroenterol Hepatol 6(3):27582

7. Lowenfels AB, Maisonneuve P, DiMagno EP et al (1997) Hereditary pancreatitis and the risk of pancreatic cancer. International Hereditary Pancreatitis Study Group. J Natl Cancer Inst 89(6):442-6

8. Giardiello FM, Brensinger JD, Tersmette AC et al (2000) Very high risk of cancer in familial Peutz-Jeghers syndrome. Gastroenterology 119(6):1447-53

9. Yoon KH, Lee JH, Kim JW et al (2006) Epidemic obesity and type 2 diabetes in Asia. Lancet 368(9548):1681-8

10. Muniraj T, Chari ST (2012) Diabetes and pancreatic cancer. Minerva Gastroenterol Dietol 58(4):331-345

11. Huxley R, Ansary-Moghaddam A, Berrington de González A, Barzi F, Woodward M (2005) Type-II diabetes and pancreatic cancer: a meta-analysis of 36 studies. Br J Cancer 92(11):2076-83

12. Li D (2012) Diabetes and pancreatic cancer. Mol Carcinog 51(1): 64-74

13. Howes N, Neoptolemos JP (2002) Risk of pancreatic ductal adenocarcinoma in chronic pancreatitis. Gut 51(6):765-76

14. Chari ST, Mohan V, Pitchumoni CS et al (1994) Risk of pancreatic carcinoma in tropical calcifying pancreatitis: an epidemiologic study. Pancreas 9(1):62-66

15. Rebours V, Boutron-Ruault MC, Schnee M et al (2008) Risk of pancreatic adenocarcinoma in patients with hereditary pancreatitis: a national exhaustive series. Am J Gastroenterol 103(1):111-9

16. Bansal P, Sonnenberg A (1995) Pancreatitis is a risk factor for pancreatic cancer. Gastroenterology 109(1):247-51

17. Iodice S, Gandini S, Maisonneuve P, Lowenfels AB (2008) Tobacco and the risk of pancreatic cancer: a review and meta-analysis. Langenbecks Arch Surg 393(4):535-45

18. Bosetti C, Lucenteforte E, Silverman DT et al (2012) Cigarette smoking and pancreatic cancer: an analysis from the International Pancreatic Cancer Case-control Consortium (Panc4). Ann Oncol 23:1880-8

19. Boffetta P, Hecht S, Gray N, Gupta P, Straif K (2008) Smokeless tobacco and cancer. Lancet Oncol 9(7):667-75

20. Jang JH, Cotterchio M, Borgida A et al (2012) Genetic variants in carcinogen-metabolizing enzymes, cigarette smoking and pancreatic cancer risk. Carcinogenesis 33(4):818-27
21. Pandol SJ, Apte MV, Wilson JS et al (2012) The burning question: why is smoking a risk factor for pancreatic cancer? Pancreatology 12(4):344-9

22. Raimondi S, Maisonneuve P, Löhr J-M, Lowenfels AB (2007) Early onset pancreatic cancer: evidence of a major role for smoking and genetic factors. Cancer Epidemiol Biomarkers Prev 16(9): 1894-7

23. Yadav D, Lowenfels AB (2013) The epidemiology of pancreatitis and pancreatic cancer. Gastroenterol 144(6):1252-1261

24. Kreiger N, Lacroix J, Sloan M (2001) Hormonal factors and pancreatic cancer in women. Ann Epidemiol 11(8):563-7

25. Silverman DT, Hoover RN, Brown LM et al (2003) Why do Black Americans have a higher risk of pancreatic cancer than White Americans? Epidemiology 14(1):45-54

26. Ye W, Lagergren J, Weiderpass E et al (2002) Alcohol abuse and the risk of pancreatic cancer. Gut 51:236-9

27. Jiao L, Silverman DT, Schairer C et al (2009) Alcohol use and risk of pancreatic cancer: the NIH-AARP Diet and Health Study. Am J Epidemiol 169(9):1043-51

28. Vrieling A, Verhage BAJ, van Duijnhoven FJB et al (2009) Fruit and vegetable consumption and pancreatic cancer risk in the European Prospective Investigation into Cancer and Nutrition. Int J Cancer 124(8):1926-34

29. Ghorbani Z, Hekmatdoost A, Zinab HE et al (2015) Dietary food groups intake and cooking methods associations with pancreatic cancer: a case-control study. Indian J Gastroenterol 34(3):225-32

30. Stolzenberg-Solomon RZ, Pietinen P, Taylor PR, Virtamo J, Albanes D (2002) Prospective study of diet and pancreatic cancer in male smokers. Am J Epidemiol 155:783-92

31. Lee IM, Sesso HD, Oguma Y, Paffenbarger RS Jr (2003) Physical activity, bodyweight, and pancreatic cancer mortality. Br J Cancer 88:679-83

32. Bonifazi M, Gallus S, Bosetti C et al (2010) Aspirin use and pancreatic cancer risk. Eur J Cancer Prev 19:352-4

33. Streicher SA, Yu H, Lu L, Kidd MS, Risch HA (2014) Case-control study of aspirin use and risk of pancreatic cancer. Cancer Epidemiol Biomarkers Prev 23(7):1254-63. doi:10.1158/1055-9965.EPI-131284

34. Tan XL, Reid Lombardo KM, Bamlet WR et al (2011) Aspirin, nonsteroidal anti-inflammatory drugs, acetaminophen, and pancreatic cancer risk: a clinic-based case-control study. Cancer Prev Res 4:1835-41

35. Khurana V, Sheth A, Caldito G, Barkin JS (2007) Statins reduce the risk of pancreatic cancer in humans: a case-control study of half a million veterans. Pancreas 34(2):260-5 